\title{
From free jets to clinging wall jets: The influence of a horizontal boundary on a horizontally forced buoyant jet
}

\author{
H. C. Burridge ${ }^{1, *}$ and G. R. Hunt ${ }^{2}$ \\ ${ }^{1}$ Department of Civil and Environmental Engineering, Imperial College London, Skempton Building, \\ South Kensington Campus, London SW7 2AZ, United Kingdom \\ ${ }^{2}$ Department of Engineering, University of Cambridge, Cambridge CB2 1PZ, United Kingdom
}

(Received 3 May 2016; published 8 February 2017)

\begin{abstract}
We investigate the incompressible turbulent jet formed when buoyant fluid is steadily ejected horizontally from a circular source into an otherwise quiescent uniform environment. As our primary focus, we introduce a horizontal boundary beneath the source. For sufficiently small separations, the jet attaches and clings to the boundary, herein the "clinging jet," before, farther downstream, the jet is pulled away from the boundary by the buoyancy force. For larger source-boundary separations, the buoyant jet is free to rise under the action of the buoyancy force, herein the "free jet." Based on measurements of saline jets in freshwater surroundings we deduce the conditions required for a jet to cling. We present a data set that spans a broad range of source conditions for the variation in volume flux (indicative of entrainment), jet perimeter, and jet centerline for both "clinging" and "free" jets. For source Froude numbers $\mathrm{Fr}_{0} \geqslant 12$ the data collapse when scaled, displaying universal behaviors for both clinging and free jets. Our results for the variation in the volume flux across horizontal planes, $\pi Q_{j e t}$, show that within a few jet lengths of the source, $\pi Q_{j e t}$ for the clinging jet exceeds that of a free jet with identical source conditions. However, when examined in a coordinate following the jet centerline $\pi Q_{j e t}$ for free jets is greater. Finally, we propose a new parametrization for an existing integral model which agrees well with our experimental data as well as with data from other studies. Our findings offer the potential to tailor the dilution of horizontal buoyant jets by altering the distance at which they are released from a boundary.
\end{abstract}

DOI: 10.1103/PhysRevFluids.2.023501

\section{INTRODUCTION}

Remarkably, the fundamental flow associated with a horizontal buoyant release from a circular source, of radius $r_{0}$, that attaches and clings to a horizontal planar boundary has received relatively little attention. Herein our focus is on steady high Reynolds number releases of fluid, of density $\rho_{0}$, into quiescent uniform surroundings of density $\rho_{e} \neq \rho_{0}$. We restrict our attention to localized releases of miscible fluids that are Boussinesq, i.e., incompressible flows for which $\left|\rho_{0}-\rho_{e}\right| \ll \rho_{e}$, and so our findings and conclusions (Sec. VI) apply equally to both positively and negatively buoyant jets whose densities differ only marginally from their surroundings. For sufficiently forced releases these conditions establish a predominantly horizontal jet-like turbulent flow near to the source. Farther from the source, the trajectory of the flow curves as a consequence of the buoyancy force [Fig. 1(a)] and far from the source the flow becomes plume-like. As for non-buoyant jets $[1,2]$ and vertical plumes [3-5], entrainment of ambient fluid and the resulting mixing and dilution of the source fluid are defining features of these so-called horizontal buoyant jets. The aforementioned mixing is instrumental to a number of industrial applications [6].

When a rigid horizontal boundary is placed close to the source of an otherwise free jet [Fig. 1(a)] the flow established may be altered significantly [Fig. 1(b)]. Herein we consider a smooth impermeable planar boundary, positioned below (or above) the source of a positively (or

*h.burridge@imperial.ac.uk 

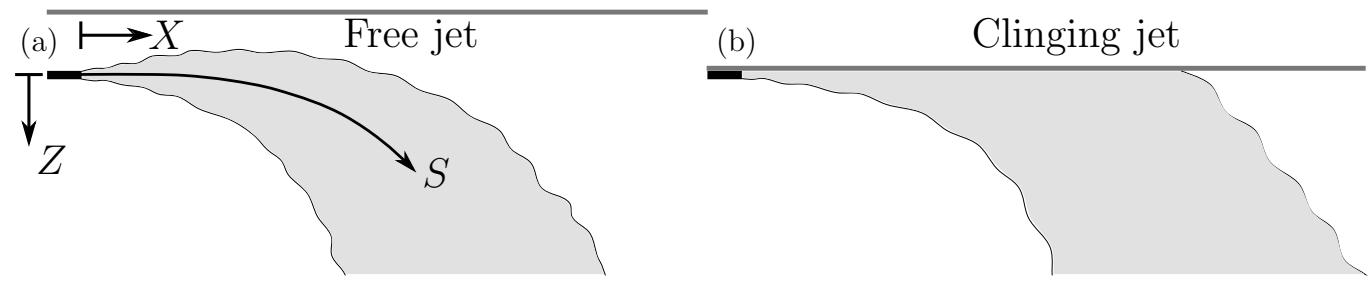

FIG. 1. Schematic illustrations of (a) a dense free jet and (b) a dense clinging jet. The ejected fluid is shaded gray with the bounding envelope (jet perimeter) marked by black lines. The horizontal boundary is marked as a dark gray line above the jet source.

negatively) buoyant jet. As depicted in Fig. 1(b), the jet may attach to (or flow along) the boundary, behavior sometimes referred to as the Coandă effect, and cling to the boundary for a distance before the (vertical) buoyancy force pulls the jet away. We refer to the resulting flow as a "clinging jet" (cf. a wall jet in the non-buoyant case $\rho_{0}=\rho_{e}$; e.g., Refs. [7,8]). By contrast, when the vertical distance between the boundary and the source is sufficiently large that the boundary has no discernible influence on the behavior of the jet, we refer to the resulting flow as a horizontal buoyant "free jet." While there is no doubt that the attachment of a jet to a boundary falls under the widespread usage of the term "Coandă effect," modern usage encompasses a number of distinct physical mechanisms, each of which may contribute to the surface attachment of a flow in any given circumstance [9]. We therefore choose to avoid further use of the term.

We investigate, by experiment, the mixing and entrainment of both clinging and free jets, through an examination of the spatial variation in volume flux across horizontal planes. Our results for the free jet provide a reference case for comparison with our primary focus, the clinging jet. When examined in a vertical coordinate $Z$ [Fig. 1(a)], our measurements show that over moderate vertical extents, the volume flux entrained by clinging jets exceeds that by free jets, suggesting the presence of the boundary increases the entrainment. Physical reasoning for this result is provided by examination of jet trajectories and bounding flow envelopes for which we establish a coordinate $S$ following the centerline of the jet [Fig. 1(a)], a coordinate in which the volume flux in clinging jets is always less than in free jets. This leads us to deduce that the role of the boundary on the jet is twofold: (1) it locally restricts entrainment and (2) it diverts the trajectory, keeping the jet closer to the horizontal plane of the boundary for a greater distance. The extent of the diversion is such that clinging jets travel farther than free jets (per unit vertical distance) so that in a vertical coordinate, the clinging jet entrains a greater volume flux, a result not obvious a priori. The bounding flow envelopes allow us to quantify directly the influence of the horizontal boundary on jet trajectory. For suitably forced releases (which we find require the source Froude number to exceed approximately 12 for the high Reynolds number releases considered), we hypothesize that both free jets and clinging jets have universal dimensionless forms, regardless of the vertical separation between source and boundary. Suitably nondimensionalized, our results (recorded over a broad range of source conditions) collapse to a single behavior for free jets and for clinging jets, thereby confirming this hypothesis and showing our results to be valid for all sufficiently forced horizontal turbulent buoyant jets.

The remainder of the paper is structured as follows. First, in Sec. II we present a brief overview of the background literature and place our work in context. An overview of the dimensional considerations and details of the experimental setup devised to test our hypothesis are then given in Secs. III and IV, respectively. In Sec. V A we present measurements of the volume fluxes across horizontal planes in free and clinging jets and propose a new parametrization for the fluxes in free jets. An extension, in the form of a simple coordinate mapping, provides the corresponding parametrization for clinging jets. From measurements of the flow envelopes and of scalar concentrations, we establish estimates for the trajectory of the jet centerline (Sec. V B) and deduce a quantitative measure for the extent of the deflection in the clinging jet trajectory. Coupling our measurements of volume flux and these centerline trajectories, we examine the entrainment per 
unit distance along the centerline (Sec. V C). We discuss our findings and draw our conclusions in Sec. VI.

\section{BACKGROUND AND CONTEXT}

The advancement in the fundamental understanding of vertical buoyant jets and plumes that the mid-20th century provided [3,4,10] was soon applied to horizontal buoyant jets [11,12]. Due to their application to discharges of waste water into the ocean, the laboratory and theoretical modeling of horizontal buoyant jets sought to incorporate the effects of an ambient stratification and a flowing current at an early stage [13]. Indeed, a strong focus on these aspects has continued [14,15], maintained by their relevance to a number of applications, including to building ventilation systems where jets may be used to heat or cool, and to environmental concerns, including the fate of pollutants released from car exhausts [16].

The potential for discharges into the oceans to pollute gives rise to a natural interest in their environmental and ecological impact; the impact of a given discharge inherently depending on its location, dilution, and mixing rates. Research has gone some way to providing insights for answering such questions but developing an understanding of the full problem is nontrivial. Even for the simplified case of a horizontal buoyant free jet in a quiescent uniform ambient, examination of the existing data for the location of the jet centerline [17-22] and jet dilution [20-22] reveals a relatively wide spread of results. Alongside enhancing our understanding of clinging jets, an objective of this study is to provide an extensive dataset for free jets in order to enable comparisons with these existing datasets.

In a number of applications the development of the jet is affected by physical constraints, for example, the confines of the room being warmed by a fan heater, the heater producing the buoyant jet. Another example is an outfall discharge located relatively close to shore where the water depth may be regarded as shallow [23]. Sobey et al. [19] examined the role of the free surface on the dynamics of positively buoyant horizontal releases, for releases made both near and far from a horizontal bottom boundary representing the bed. Their measurements of the trajectory of the jets are insightful and, despite the scatter, show a broad agreement with our measurements. However, clear differences between releases near to and far from the bottom boundary, i.e., clinging and free jets, were not evident in their measurements of dilution. Our current study is intended to provide such additional insights.

Studies of horizontal buoyant clinging jets originating from circular sources are relatively limited. The focus of theoretical studies has been on the two-dimensional planar analogue [24], presumably since attachment to a (planar) surface by buoyant jets from circular horizontal sources lacks any obvious symmetry. Sharp and Vyas [25] provide a notable exception, studying clinging jets from circular sources with both experiments and a predictive integral model which, after a zone of flow establishment, considers the flow to be comprised of two regions. The first, an attached region, incorporates the effects of friction between the boundary and the clinging jet and assumes the cross-stream section of the jet forms a segment of a circle. In the second region, an integral model quantifies the fluxes within the jet rising away from the horizontal boundary. Sharp and Vyas [25] present measurements for jet trajectories, dilution rates, and the lateral extent over which the jet clings to the boundary, hereinafter the cling length $X_{c}$, deducing that this length scales on the jet length.

\section{DIMENSIONAL CONSIDERATIONS}

We restrict our attention to releases where (1) the forcing at the source is sufficiently large so that inertial forces dominate the buoyancy force at the source and (2) the flow established is turbulent, i.e., viscous forces are small relative to the inertia of the flow. The source fluxes of (specific) momentum, $\pi M_{0}$, and buoyancy, $\pi B_{0}$, then characterize the source. For the near-field region, the resulting dominant length scale (the jet length) and the dominant volume flux scale may be written 
(e.g., Ref. [26])

$$
L_{M}=\frac{M_{0}^{3 / 4}}{B_{0}^{1 / 2}} \quad \text { and } \quad Q_{M}=\frac{M_{0}^{5 / 4}}{B_{0}^{1 / 2}},
$$

respectively, where the subscript $M$ is used to convey that it is the source momentum flux that is dominant in this region of flow. Consideration of the ratio of inertia to buoyancy forces at the source establishes the source Froude number as the controlling parameter

$$
\mathrm{Fr}_{0}=\frac{V_{0}}{\sqrt{r_{0} g_{0}^{\prime}}}=\frac{M_{0}^{5 / 4}}{Q_{0} B_{0}^{1 / 2}}=\frac{Q_{M}}{Q_{0}}=\frac{L_{M}}{r_{0}},
$$

where $V_{0}$ and $g_{0}^{\prime}=g\left|\rho_{0}-\rho_{e}\right| / \rho_{e}$ are characteristic scales for the velocity and buoyancy at the source, respectively, and $\pi Q_{0}$ is the source volume flux. In Eq. (2) and elsewhere herein we assume uniform (top-hat) profiles for the distribution of velocity and buoyancy at the source. Consideration of any other distribution merely requires the introduction of two constants for the dimensionless shape factors. Relating (1) and (2) identifies the physical scale of the source, $r_{0}=Q_{0} / M_{0}^{1 / 2}$, as an alternative length scale that is sometimes used to scale experimental data [27] and that has been identified as the dominant scale for "lazy" [5] as opposed to the relatively forced source conditions we consider herein. We note from (2) that the source scale can be expressed in terms of $\mathrm{Fr}_{0}$ and the jet length as

$$
L_{M}=r_{0} \mathrm{Fr}_{0} .
$$

Since we focus on high Froude number releases, $L_{M}$ is the natural choice of length scale.

Hereinafter we examine negatively buoyant ejections and specifically those formed by releases of saline solution in a freshwater environment. Regarding notation, our data consist of measurements of volume flux and distances, which we represent by upper-case letters when in dimensional form, e.g., $X, Z, S$, and $Q_{\text {jet }}$. When normalized by scales apparent on dimensional grounds we use lower-case letters and introduce

$$
x=X /\left(r_{0} \mathrm{Fr}_{0}\right), \quad z=Z /\left(r_{0} \mathrm{Fr}_{0}\right), \quad s=S /\left(r_{0} \mathrm{Fr}_{0}\right), \quad \text { and } \quad q_{\text {jet }}=Q_{\text {jet }} /\left(Q_{0} \operatorname{Fr}_{0}\right) .
$$

Despite the wide occurrence of jets in stratified environments, or where a current or other background flow is present, our analysis (see Appendix A) indicates that it is not always necessary to consider these effects directly in order to capture the bulk dynamics of the jet, at least moderately close to the source. Therein we take a discharge into the ocean and the warming of a room by a fan heater as example applications where the environment is typically stratified to show that the dynamics of jets in otherwise uniform quiescent environments (as considered herein) can provide useful insights over moderate spatial extents.

\section{EXPERIMENTS}

\section{A. Experimental setup}

Figure 2 depicts our experimental setup. Negatively buoyant saline jets were formed by injecting horizontally a coloured solution of sodium chloride $(\mathrm{NaCl})$ from a circular source into a clear Perspex visualization tank of square horizontal cross section (sides of length $100 \mathrm{~cm}$ ) that was filled with fresh water to a depth of $75 \mathrm{~cm}$. Sources with exit radii $r_{0}=0.138,0.299$, and $0.593 \mathrm{~cm}$, measured to $\pm 0.001 \mathrm{~cm}$, were tested. The boundary consisted of a square Perspex sheet $(90 \times 90 \mathrm{~cm}$ and thickness $1 \mathrm{~cm}$ ) held rigidly $10 \mathrm{~cm}$ (approximately) beneath the free surface. The boundary was carefully leveled and positioned with one edge resting centrally against one vertical wall of the tank. Thus fresh water was free to pass around the boundary through a "U-shaped" horizontal section of area $2800 \mathrm{~cm}^{2}$. The source was positioned centrally on the lower surface of the boundary, as depicted in Fig. 2, by two threaded stainless steel bars $(0.6 \mathrm{~cm}$ diameter $)$. These bars enabled fine adjustment 


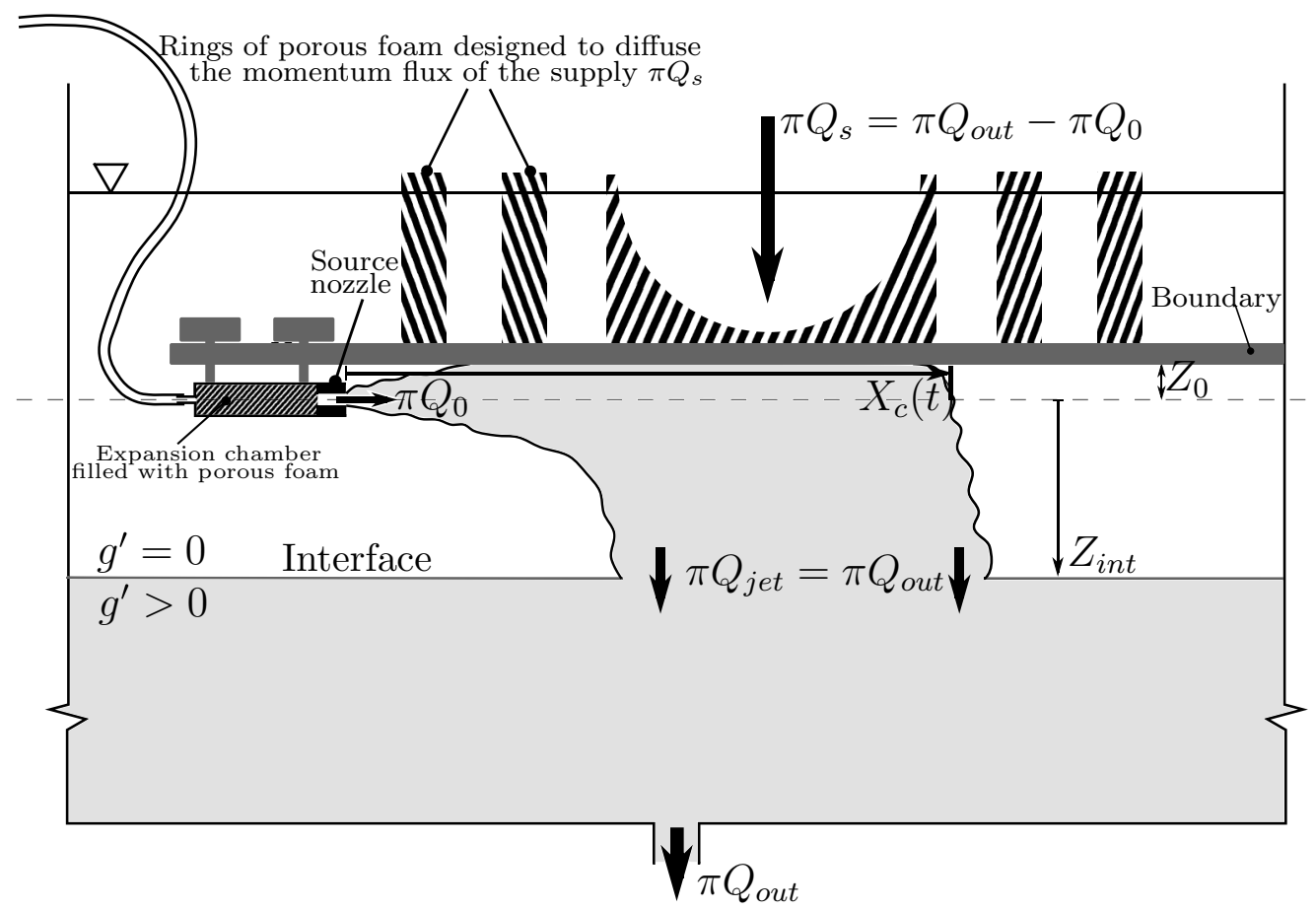

FIG. 2. Illustration of the experimental setup used to measure the volume flux across a horizontal plane, $\pi Q_{j e t}$, in a saline jet at a distance $Z_{\text {int }}$ below the source. A circular nozzle positioned a distance $Z_{0}$ below the boundary steadily ejected saline solution (shaded light gray) at a rate $\pi Q_{0}\left(\mathrm{~m}^{3} \mathrm{~s}^{-1}\right)$ within a visualization tank initially filled with fresh water. A known volume flux $\pi Q_{\text {out }}$ is extracted from the base of the tank and fresh water supplied (above the boundary) at a rate $\pi Q_{s}=\pi Q_{\text {out }}-\pi Q_{0}\left(\mathrm{~m}^{3} \mathrm{~s}^{-1}\right)$. The source-boundary separation $Z_{0}$ determined whether a free jet or a clinging jet formed (see Sec. IVE). The schematic depicts a clinging jet which has established a steady two-layer stratification with interface at $Z=Z_{\text {int }}$. The instantaneous cling length of the jet, $X_{c}(t)$, is marked.

of the nozzle orientation and position, to ensure it sat horizontally and at a set distance $Z_{0}(\mathrm{~cm})$ between the lower surface of the boundary and the source centerline.

The nozzle was designed with an internal expansion chamber which was filled with porous foam to excite turbulence in the flow prior to ejection. In addition, the steady source flowrate was set sufficiently high to ensure the jet was visibly turbulent close to the source (always within $2 r_{0}$ downstream of the exit plane). The flow rate was set using an Ismatec MCP-Z Process gear pump and measured to an accuracy of $0.2 \%$ by an Apollo Lowflo Pelton wheel flowmeter RN3-1850 and 202Di rate totalizer. The flowrates were varied between experiments, $5.5 \mathrm{~cm}^{3} / \mathrm{s} \leqslant \pi Q_{0} \leqslant 17 \mathrm{~cm}^{3} / \mathrm{s}$, to give source velocity estimates of $44.0 \mathrm{~cm} / \mathrm{s} \leqslant V_{0}=Q_{0} / r_{0}^{2} \leqslant 149.0 \mathrm{~cm} / \mathrm{s}$. The resulting source Reynolds numbers were $2650 \leqslant \operatorname{Re}_{0}=V_{0} r_{0} / v \leqslant 10510$ ( $v$ denoting the kinematic viscosity of the source fluid). For the vast majority of experiments $\operatorname{Re}_{0} \gtrsim 5000$.

The density of the source saline and of the fresh water in the tank were measured to an accuracy of $0.0005 \%$ using an Anton Paar DMA 5000M densitometer. The resulting source buoyancy was calculated and systematically varied between experiments within the range $13.0 \mathrm{~cm} / \mathrm{s}^{2} \leqslant g_{0}^{\prime} \leqslant$ $160.0 \mathrm{~cm} / \mathrm{s}^{2}$. The source Froude numbers (known to an uncertainty of approximately $3 \%$ ) were thereby in the range $4.4 \leqslant \mathrm{Fr}_{0} \leqslant 81.7$; the upper limit was governed by our equipment. As we show in Sec. V, our scaled data collapse for $\mathrm{Fr}_{0} \geqslant 12.0$. We exclude data obtained for $\mathrm{Fr}_{0}<12.0$ as evidently at lower Froude number the jet length can no longer be considered as the single dominant 
length scale of the flow; similar transitions occur for other freely entraining turbulent buoyant flows, e.g., plumes [5] and fountains [28].

\section{B. Measurement of volume flux}

Measurements of volume flux were obtained in different horizontal planes using a technique originally conceived by Baines [29] for inferring the volume flux in a turbulent plume. The basic principle is to impose a weak vertical (downward) flow in the freshwater environment within which the plume or, in our case, the horizontal saline jet develops and to record the level at which a stationary density interface forms (Fig. 2). The weak vertical flow was achieved by extracting a prescribed volume flux, $\pi Q_{\text {out }}$, from the base of the tank while keeping the total liquid depth constant by providing a continuous (low momentum) supply of fresh water near the surface. Once the interface is stationary, the volume flux in the jet crossing the horizontal interface $\pi Q_{j e t}=\pi Q_{\text {out }}$, the latter being a known prescribed value. Recording the level of the stationary interface $Z=Z_{\text {int }}$ (in our case to accuracies varying from $\pm 0.2 \mathrm{~cm}$ to $\pm 1.5 \mathrm{~cm}$ or approximately $1 \%$ to $6 \%$ depending of the position of the interface and the scale of any waves that formed at the interface) thereby provides a direct measurement of the volume flux in the jet crossing a horizontal plane at a vertical distance $Z_{\text {int }}$ from the source. By iteratively adjusting $\pi Q_{\text {out }}$ and allowing time for the interface to adjust to a new stationary level, the volume flux $\pi Q_{j e t}$ can be estimated over a range of heights. The interface become stationary between 900 and $3000 \mathrm{~s}$ after iterative adjustments of $\pi Q_{\text {out }}$.

The extracted volume flux was driven by a Pompe Cucchi N1 gear metering pump and measured with an Apollo Flow RN3/20/5 turbine flowmeter, which, with an external servo-cooling unit, provided flow rates between $85.0 \mathrm{~cm}^{3} / \mathrm{s} \leqslant \pi Q_{\text {out }} \leqslant 540.0 \mathrm{~cm}^{3} / \mathrm{s}$, measured to accuracies within $0.3 \%$. The resulting imposed flow in the ambient could, justifiably, be regarded as weak since the maximum ambient velocities (in the plane of the boundary) were small (never exceeding $0.008 V_{0}$ ) compared with the inertial velocity scale at the source $V_{0}$. Background velocities in the ambient at other heights never exceeded $0.002 V_{0}$.

The well-established "Baines method" enables repeatable measurement of volume flux across any given horizontal plane (the interface) of a free or a clinging jet, simply by varying the rate of outflow from the tank base $\pi Q_{o u t}$. It is worth noting that the standard measure of volume flux in a buoyant jet is that made locally in a plane perpendicular to the jet centerline. Due to the action of the buoyancy force, the vertical momentum flux increases (from a value of zero at the source) with increasing vertical distance from the source, so that asymptotically far from the source the flow tends to the vertical; in other words, the measurement of volume flux in a horizontal plane provides a direct measure of the true volume flux of the jet.

By contrast, relatively close to the source, the horizontal momentum flux is significant compared to the vertical momentum flux (we find for $z \lesssim 1.5$; see Sec. VA), and thus measurements of volume flux made in horizontal planes may be expected to differ from measurements made locally perpendicular to the jet centerline. Crucially, in this near-field region, the flow is expected to be jet-like despite the presence of buoyancy. Indeed, this expectation is supported by our own measurements (Sec. V C), which indicate that the volume flux in a horizontally ejected dense saline jet increases linearly with distance along the centerline, i.e., as it does for a classic pure jet [26]. This close agreement between our measurement of the volume flux in horizontal planes $\pi Q_{j e t}$ and the classic linear scaling suggests that, in this near-field region, the volume flux in the buoyant jet varies only marginally with the angle between the centerline and the (horizontal) plane of measurement. Notably, comparison of our measurements with those reported in complementary studies [20-22] confirms that our experimental technique did not introduce any significant bias to the measurements of the volume flux $\pi Q_{j e t}$; see Sec. V A 1 .

A number of alternate experimental techniques to measure the jet volume flux were available to us, each with its own limitations. For example, volume flux estimates could have been obtained on interpolating discrete measurements obtained from an array of hot-wire anemometers mounted on a grid placed in the flow. Implementing this approach presents significant challenges, notably as the 
(a)

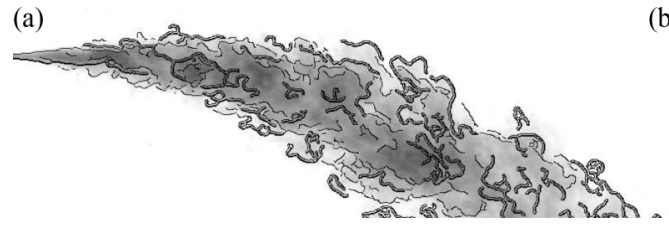

(b)

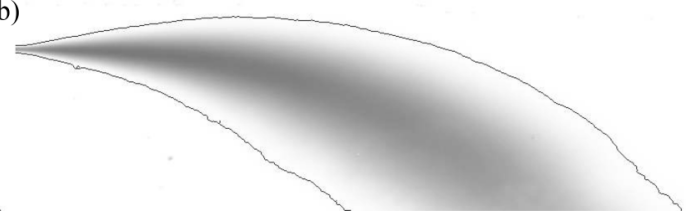

FIG. 3. A free jet with source condition $\mathrm{Fr}_{0}=47.3, r_{0}=0.1375 \mathrm{~cm}$. (a) Instantaneous image with edges highlighted. (b) Time-averaged image with flow envelope overlaid.

grid would need to be positioned perpendicular to the jet centerline (the orientation of the centerline typically not known a priori), and as a result this approach is expected to introduce fundamental uncertainties. Moreover, the lack of symmetry in the flow under examination renders any planar measurement unsuitable for obtaining measurements of volume flux, e.g., standard particle image velocimitry or planar light-induced fluorescence.

\section{Acquisition of flow envelopes and ensemble averaging}

The visualisation tank was diffusely back-lit using an array of high-frequency fluorescent tubes. The source saline was stained using methylene blue so that the light attenuated at any spatial location corresponded to the local dye concentration [30,31]. Images of the interface and jet were digitally recorded using a CCD camera (a JAI CVM $\left.4{ }^{+} \mathrm{CL}\right)$ at a frequency of 12 frames per second. Interrogation of the images showing the interface provided confirmation that it had reached a stationary height. Furthermore, time averaging and interrogation of the gradients of light intensity of the jet images allowed the mean centerline and mean flow envelopes to be determined (Fig. 3). For details refer to Appendix B.

In order to ensemble average images across all source conditions we applied an estimate for the horizontal offset required in order to colocate the virtual origin of each source condition (a vertical offset is not required since the flow is only forced horizontally at the source). The suitability of a horizontal offset based on a virtual origin correction that traces the far-field behavior back to a point is questionable since the far-field flow asymptotes to both $z \rightarrow \infty$ and $x \rightarrow \infty$. However, we note that for the high-Fr $r_{0}$ jets of interest here, the near-field spreading rate is $\tan ^{-1}\left(2 \alpha_{j}\right)$ [32], where $\alpha_{j}$ denotes the top-hat entrainment coefficient for a jet. Moreover, horizontal momentum flux is conserved as for a horizontal pure jet. As such, we assumed the flow to be jet-like near the source and applied the following simple geometric virtual origin offset horizontally:

$$
X_{v o}=\frac{r_{0}}{2 \alpha_{j}}
$$

In Eq. (5) we take $\alpha_{j}=0.055 \sqrt{2}$; see Ref. [26]. For our experiments this resulted in $0.01 \leqslant$ $X_{v o} /\left(r_{0} \mathrm{Fr}_{0}\right) \leqslant 0.2$.

\section{Measuring the length of attachment for clinging jets}

Clinging jets remained attached to the boundary until the buoyancy force dominated and pulled the dense fluid away. We denote the maximum horizontal distance between the source and the location of the point at which the jet detached from the boundary as the instantaneous cling length, $X_{c}(t)$. For each experiment, the instantaneous cling length (Fig. 2) was time-averaged to provide an estimate of the mean cling length, $\overline{X_{c}}$. The uncertainties in our measurements of $\overline{X_{c}}$ were between $3 \%$ and $8 \%$ and were due in part to the wide fluctuations in cling length observed as large-scale eddies "pulsed" through (see Ref. [25]) and to difficulties in detecting the (dilute) fluid farthest from the source. 


\section{E. Forming free jets and clinging jets}

Whether the flow established could be regarded, or classified, as a free jet or a clinging jet depended solely on the distance between the source and the boundary relative to the jet length. To classify as a free jet we required that the boundary had no observable effect on the flow established, i.e., the flow was indistinguishable from that formed in the absence of the boundary. To classify as a clinging jet we required that not only was the otherwise free jet flow affected by the presence of the boundary but, crucially, that further decreases in source-boundary separation did not result in any measurable changes to the dynamics of the jet (see Ref. [19]). Regarding the latter, specifically, we note that for intermediate source-boundary separations the attachment was intermittent and the evolution of the jet depended on the precise value of the source-boundary separation, relative to the jet length; the behavior of such jets is beyond the scope of the current investigation. For smaller separations the intermittency was absent and (suitably scaled) a universal form of behavior established.

For sufficiently large dimensionless source-boundary separations $\left[z_{0}=Z_{0} /\left(r_{0} \mathrm{Fr}_{0}\right)\right]$ the uppermost edge of the jet was always far enough from the boundary that its presence had no observable impact on the formation of the jet, which was then deemed "free." Free jets were observed for $z_{0} \gtrsim 0.7$. For free jets, the point of minimum separation (at a horizontal location of $x \approx 2$ ) between the uppermost edge of the jet and the boundary was approximately equal to the local half width of the jet (see Fig. 7 for an illustration).

On decreasing the source-boundary separation below $z_{0} \approx 0.7$ the flow became increasingly affected by the presence of the boundary, and the jet volume fluxes and trajectories changed measurably with separation. In some cases the jet was observed to attach and detach periodically. For separations of $z_{0} \lesssim 0.2$, no variation in the (dimensionless) volume fluxes or trajectories could be observed or measured. Our data indicate that free jets form for $z_{0} \gtrsim 0.7$ and clinging jets for $z_{0} \lesssim 0.2$; data obtained for intermediate source-boundary separations, namely, $0.2<z_{0}<0.7$, have been omitted.

\section{RESULTS AND DISCUSSION}

\section{A. Entrainment and the vertical variation in volume flux}

Our measurements of volume flux are presented in Fig. 4. The figure shows the variation in the data with the vertical coordinate, as is consistent with our measurement technique and as is typically reported in other studies utilising other measurement techniques [22]. The unscaled data [Fig. 4(a)] indicate that volume flux increases with vertical distance from the source, although no apparent bias is evident between measurements for free and clinging jets. When scaled [Fig. 4(b)], the data for free jets collapse to a single universal behavior; likewise the data for clinging jets. However, distinct differences between the universal curves are evident. Most strikingly, for identical source conditions, a clinging jet has a greater volume flux than a free jet for $z \lesssim 3$, while still farther from the source the volume fluxes in free and clinging jets are approximately equal. This result suggests that over the same vertical extent clinging jets entrain more than free jets, a finding which at first sight may appear counterintuitive. Instead, one might have anticipated that the boundary would have provided a natural restriction to the entrainment of clinging jets. Our measurements of volume flux compare well with those inferred from datasets reported elsewhere (see Fig. 5). Further investigation of and physical reasoning for this result is provided in Sec. V C.

\section{A parametrization for free jets}

A model for predicting the volume fluxes and trajectories of buoyant jets ejected at various angles (including horizontally) is presented by Lane-Serff et al. [32], who integrated the conservation equations for the horizontal and vertical fluxes of volume, momentum, and buoyancy. Their simplifying assumptions were that the distributions of velocity and buoyancy are self-similar (with characteristic half-widths $b$ and $\lambda b$, respectively). In addition, they invoke the entrainment 
FROM FREE JETS TO CLINGING WALL JETS: THE ...
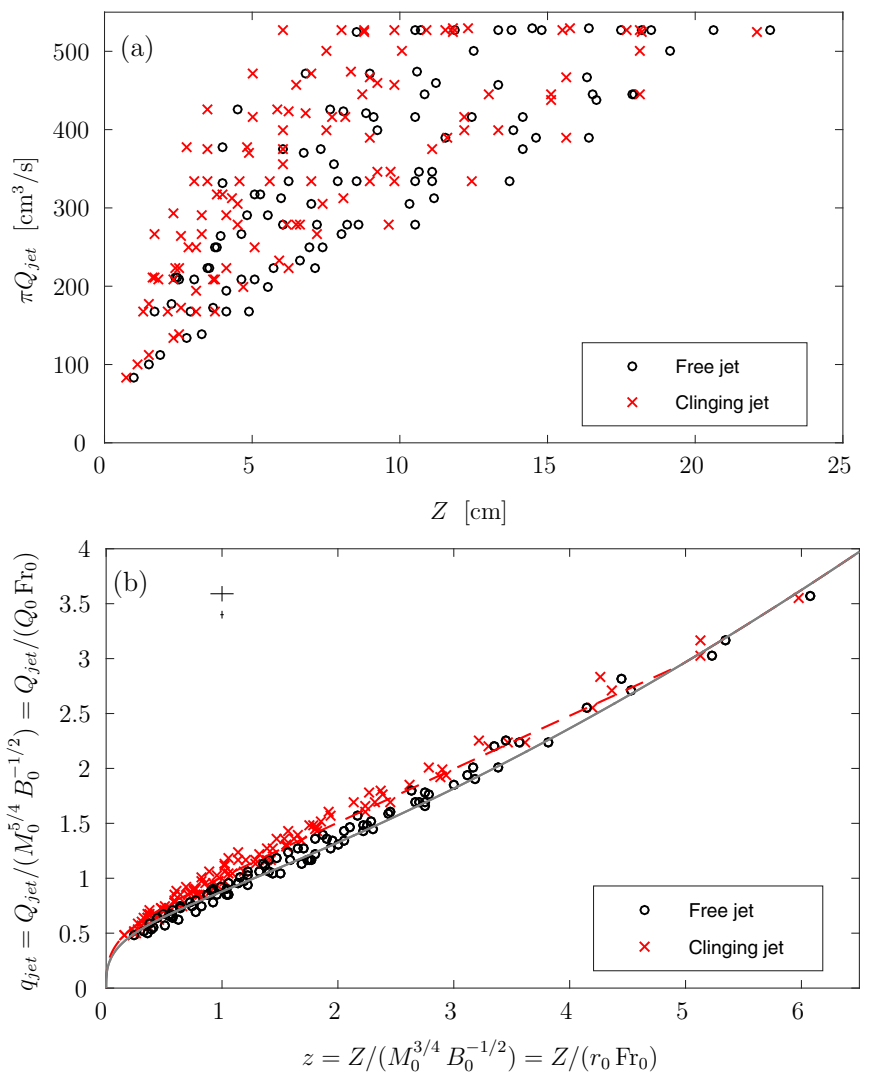

FIG. 4. (a) Volume flux $\pi Q_{j e t}$ variation with height $Z$. (b) The scaled data $Q_{j e t} /\left(Q_{0} \mathrm{Fr}_{0}\right)$ with $Z /\left(r_{0} \mathrm{Fr}_{0}\right)$. In (b) predictions for free jets (based on the model of Lane-Serff et al. [32] with the parametrization discussed in Sec. V A 1) are marked by the gray solid line. Predictions for clinging jets (Sec. V A 1) are marked by the dashed red line. Error bars associated with the maximum and minimum uncertainties from any given experiment are plotted at $(x=1, z \approx 3.5)$.

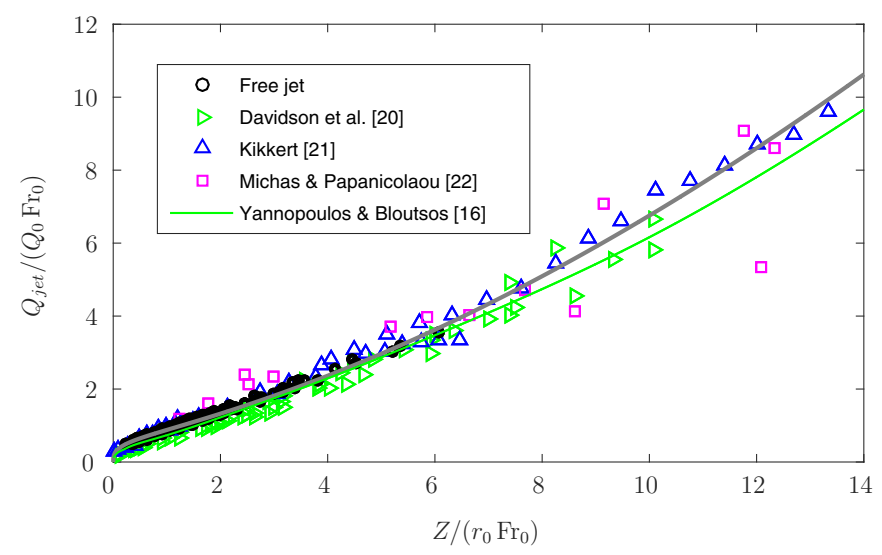

FIG. 5. Variation in volume flux, $Q_{j e t} /\left(Q_{0} \mathrm{Fr}_{0}\right)$, with height, $Z /\left(r_{0} \mathrm{Fr}_{0}\right)$, from our measurements of free jets, with the volume fluxes inferred from dilution measures in other studies as detailed in the legend. Predictions of the model of Ref. [32] with our parametrization (Sec. V A 1) is marked by the solid gray curve. The predictions from the escaping mass model of Ref. [16] are marked by the solid green curve. 
assumption [3], namely, that the entrainment velocity (locally perpendicular to the jet) is $\alpha V$, where $V$ denotes the local jet velocity in the direction of the centerline. The validity of these assumptions is more questionable for horizontal buoyant jets (particularly in the near field) than for the vertical plumes for which they were originally proposed [3]. Nonetheless, these assumptions have been shown to provide an adequate turbulence closure parametrization for a wide variety of flows [33]. Under these assumptions and those underlying classic plume theory (Boussinesq, inviscid, and incompressible flow with no mass diffusion nor swirl and neglecting the turbulent contributions to the fluxes; see Ref. [3]), Lane-Serff et al. [32] express the conservation equations in a form which can be written as

$$
\frac{\mathrm{d} Q}{\mathrm{~d} S}=2 \alpha M^{1 / 2}, \quad \frac{\mathrm{d} M_{v}}{\mathrm{~d} S}=\lambda^{2} b^{2} g^{\prime}, \quad \frac{\mathrm{d} M_{h}}{\mathrm{~d} S}=\frac{\mathrm{d} M_{0}}{\mathrm{~d} S}=0, \quad \text { and } \frac{\mathrm{d} B}{\mathrm{~d} S}=0
$$

where $\pi Q, \pi M$, and $\pi B$ are the local fluxes of volume, momentum, and buoyancy, respectively, $g^{\prime}$ the local buoyancy, and $M^{2}=M_{0}^{2}+M_{v}^{2}$ relates the local flux of momentum $M$ to its vertical component, $M_{v}$, and (conserved) horizontal component, $M_{h}=M_{0}=$ const. The conservation equations indicate that the variation in volume flux is driven by the momentum flux locally and, since $\mathrm{d} M / \mathrm{d} S=$ $\lambda^{2} b^{2} g^{\prime} \sqrt{1-\left(M_{0} / M\right)^{2}}$, the variation in the local momentum flux is determined by the relative magnitude of the source momentum flux $M_{0} / M$, the local buoyancy $g^{\prime}$ and its characteristic halfwidth $\lambda b$.

Extensions to include the second-order terms arising due to turbulent fluctuations have been made by Yannopoulos and Bloutsos [16]. Their integral model also incorporates a mechanism to encapsulate the effects of buoyant fluid rising up and "escaping" from the jet (and implicitly the flow induced in the ambient by the jet) while the jet is predominantly horizontal. Within each of the parametrizations of buoyant jets that theoretical advances have proposed, there lie a number of free parameters (just two, namely, $\alpha$ and $\lambda$ in the model of Ref. [32]). In the absence of conclusive experimental data, it is difficult to justify any particular values for these parameters or to assess the relative merits of more advanced models; for example, in our experiments no fluid was observed to "escape" from the jet. Indeed, a model that could conclusively predict the variation in volume flux and trajectories of horizontal buoyant jets would be a significant advance.

Figure 4 plots predictions based on the model of Ref. [32] with an entrainment coefficient of $\alpha=0.1 \sqrt{2}$ (recent measurements confirm this to be a reasonable value for pure plumes and forced plumes [34]). Furthermore, reasoning that the far-field flow is plume-like, there we take $\lambda_{f}=1.2$; see Refs. [35,36].

Closer to source, the flow is predominately horizontal and obviously differs from a vertical plume as the source momentum flux and the momentum flux induced by the action of the buoyancy force are nonparallel. While the Reynolds number is sufficient that the jet-ambient interface always remains unstable and turbulent, the buoyancy force adds a stabilizing effect at the upper jet-ambient interface and a destabilizing effect at the lower jet-ambient interface. Thus, the entrainment and the distribution of buoyancy within the predominantly horizontal flow is expected to be skewed (i.e., asymmetric about the centerline) and laterally constrained by the buoyancy force, an expectation qualitatively supported by the data of Ref. [37]. The lateral constraint would result in a reduced spread (variance) in the distribution of the buoyancy, and, hence, we propose to account for these near-field effects with a reduction in $\lambda$. We deem it reasonable to neglect the skewness in the distribution of the buoyancy, i.e., we parameterize the effects from the second moment of the distribution and neglect any effects from the third moment. For the high- $\mathrm{Fr}_{0}$ releases considered, the flow is jet-like in the near-field region, and one would expect the buoyancy to be advected as a passive scalar and, thereby, that the widths of the distributions of buoyancy and velocity be equal, i.e., for the near field $\lambda_{n}=1.0$. The transition from the near-field to far-field behaviors will be controlled by the ratio of the horizontal source momentum flux $M_{0}$ to the local momentum flux $M$. As such, we solve the conservation 


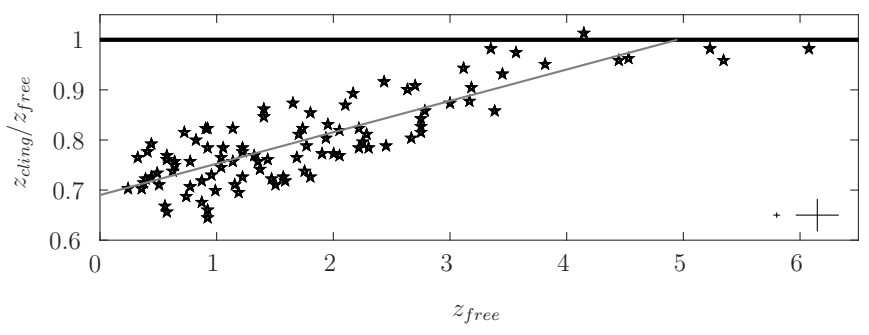

FIG. 6. The variation in the ratio of the vertical distances traveled by clinging jets and free jets to attain a given volume flux, $z_{\text {cling }} / z_{\text {free }}$, plotted against the vertical distance traveled by free jets, $z_{\text {free }}$. The horizontal line marks where clinging jets and free jets have traveled the same vertical distance to attain an identical volume flux. The sloping gray line marks the best linear fit to the data. Error bars associated with the maximum and minimum uncertainties from any given experiment are shown in the lower right-hand corner of the plot.

equations (6) taking

$$
\lambda=\lambda_{n}+\frac{M-M_{0}}{M}\left(\lambda_{f}-\lambda_{n}\right)
$$

The fit of the resulting predictions to our data is good (see Fig. 4), and this parametrization provides a better agreement with the data than can be achieved on assuming a constant value for $\lambda$.

We note that some variation between the model predictions of the fluxes across planes perpendicular to the centerline and our experimental measurements of fluxes across horizontal planes might be expected when the angle of the two planes differs significantly, which is the case close to the source. However, the good agreement between the predictions of the model and our measurements indicates that either the variations due to differences in the angle of the plane of measurement are not significant or the parametrization somehow accounts for these variations. Figure 5 shows the variation in volume flux for free jets from our measurements and that published in three other studies. Each of these three studies reports data for the variation with height of the dilution of the buoyancy measured on the centerline. By assuming that the approximate distribution of the buoyancy is Gaussian-like (broadly supported by measurements of the cross-stream profiles; e.g., Ref. [20]) the centerline buoyancy measurements, $g_{c}^{\prime}$, can be related to the uniform (cross-stream averaged) buoyancies considered in the model by $g^{\prime}=g_{c}^{\prime} / 2$. Conservation of the buoyancy flux provides $g_{0}^{\prime} Q_{0}=g^{\prime} Q_{j e t}=g_{c}^{\prime} Q_{j e t} / 2$, and, hence, we can compare the dilution measurements presented in other studies to the predictions of volume flux in our model. With only a few exceptions, all data points from the four experimental studies show good agreement with the predictions of jet volume flux from our parametrization of the model; this remains the case far farther from the source than we were able to gather data in our experiments. This agreement (for $z \lesssim 14$ ) between model predictions and the data inferred from centerline dilutions published in other studies suggest that our parametrization exhibits no significant bias due to being determined by our measurements of the fluxes across horizontal planes (for $z \lesssim 6$ ). Moreover, this agreement indicates that the variations due to differences in the angle of the plane of measurement are not significant. We may assert, therefore, that the parametrization we have determined affords a good engineering solution in terms of enabling first-order estimates of the volume flux in these complex jets.

\section{Prediction of volume flux in clinging jets}

In order to quantify the differences in entrainment between free and clinging jets, Fig. 6 plots the ratio of the vertical distance traveled to entrain a particular volume flux (we recall that our data are the volume flux crossing horizontal planes). The figure shows the variation in this ratio, $z_{\text {cling }} / z_{\text {free }}$, versus the vertical distance traveled by the free jet. The data for $z_{\text {free }} \gtrsim 4$ show the ratio reaching approximately unity, i.e., at these heights the volume fluxes in free and clinging jets 
are almost equal. At lesser heights the ratio falls substantially beneath unity, reaching values of $z_{\text {cling }} / z_{\text {free }} \approx 0.6-0.7$, with some data indicating that free jets must travel vertically $50 \%$ farther than an equivalent clinging jet to entrain the same volume flux. The data show a significant spread, consistent with approximately twice the uncertainty expected in our measurements of entrainment, with no bias in our measurements based on $\mathrm{Fr}_{0}$ being apparent in our data. However, a first-order approximation to the trend seen can be obtained on assuming a linear variation in the ratio with $z_{\text {free }}$, with a maximum value set at $z_{\text {cling }} / z_{\text {free }}=1$ (the latter to ensure that suitably far above the source the volume fluxes predicted for free are clinging jets are equal, as reflected in our data; Fig. 4). The best fit to the data (Fig. 6), for $z<5$, is

$$
z_{\text {cling }} / z_{\text {free }}=0.063 z_{\text {free }}+0.69
$$

which provides an empirical means for relating the volume fluxes in free and clinging jets. The results of this simple mapping applied to predictions of the volume flux in free jets from the model of Ref. [32] (with the parametrization (7)) are shown in Fig. 4. Despite the simplifying approximations made, this method, evidently provides a fairly good prediction of the volume flux for clinging jets.

\section{B. Envelopes and centerlines of free and clinging jets}

The universal time-averaged flow envelopes and ensemble-averaged light intensities are shown in Fig. 7 for free jets and clinging jets. The envelopes, which are those plotted from our data for $12 \leqslant \mathrm{Fr}_{0} \leqslant 82$ (with $3.0 \mathrm{~cm} \leqslant r_{0} \mathrm{Fr}_{0} \leqslant 11.5 \mathrm{~cm}$ ), show a reasonable collapse. Near the source $(x \lesssim 2)$ the envelopes for clinging jets are markedly narrower than those for free jets and are noticeably broader farther from the source $(z \gtrsim 0.6)$.

The contours of constant light intensity within the ensemble-averaged images guide our visualization of the centerlines, the centerlines having been identified by turning points in the contours of constant light intensity. Unsurprisingly the centerlines for both free and clinging jets follow the same general trend, namely, a near-field lateral projection with an increasingly downward projection. For comparison, Fig. 7 highlights points on each centerline at two fixed heights, chosen to be relatively close to, $z=0.6(\triangle)$, and far from, $z=4.0(\bigcirc)$, the source. At $z=0.6$ clinging jets have traveled approximately $30 \%$ farther $\left(s_{\text {cling }} / s_{\text {free }}=1.31\right.$ and $\left.x_{\text {cling }} / x_{\text {free }}=1.32\right)$ than free jets. This highlights the substantial diversion to the trajectory of the jet centerline that results from the attachment to the boundary. However, when at $z=4.0$, clinging jets have traveled only $13 \%$ farther (i.e., $s_{\text {cling }} / s_{\text {free }}=1.13$ ), and laterally $18 \%$ farther (i.e., $x_{\text {cling }} / x_{\text {free }}=1.18$ ). This indicates that the extension in the distance traveled by clinging jets, relative to the free jet, decreases with distance from source.

The practical application of free and clinging jets (see Sec. I) may well benefit from predictions of their volume fluxes and trajectories over the moderate vertical distances for which we report results. Our results indicate readily exploitable differences for the application of free and clinging jets. A tractable model for the prediction of the trajectories of clinging jets is not available within the literature, nor is it obvious how best to pose a simplified model. Furthermore, although the solutions to the conservation equations, (6), with the parametrization, (7), provide reasonable predictions for the vertical variation in volume flux in a free jet, the predictions of jet trajectory are not consistent with experimental measurements. However, it is not clear which physical mechanisms give rise to the inconsistencies or how best to represent these within a simplified model. It is unclear whether the inconsistencies may be resolved by, for example, varying the entrainment coefficient with the local Froude number, or accounting for the asymmetries and spatial variations in the distributions of velocity and buoyancy, or reflecting differences due to the upper and lower edges of the jet being either gravitationally stable or unstable density interfaces [16]. Highlighting which of these mechanisms are dominant is made all the less clear due to the discrepancies in the experimental datasets for the centerlines. Given these difficulties, we opt to provide descriptions of the centerlines and the envelope edges in an empirical form. Our estimates of the centerlines (obtained from contours of 

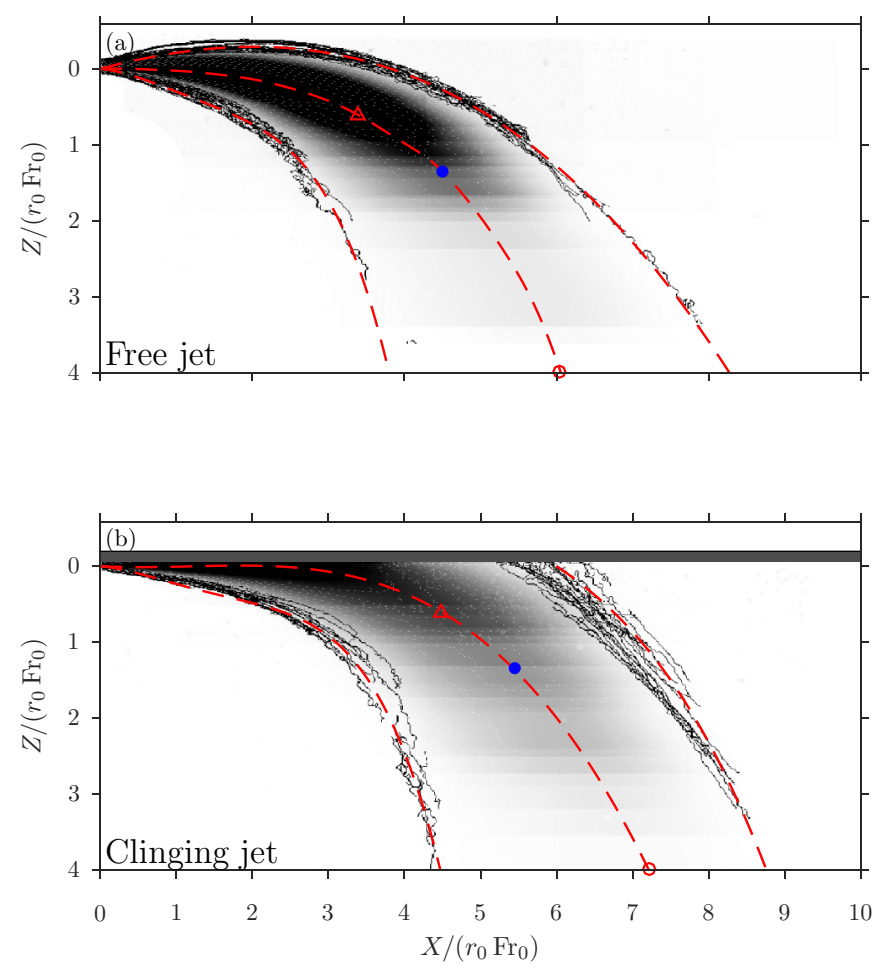

FIG. 7. Experimental measurements of the flow path taken by (a) free jets and (b) clinging jets. Flow envelopes (black lines) resulting from 46 distinct source conditions spanning $12 \leqslant \mathrm{Fr}_{0} \leqslant 82$ are shown. The gray-scale background represents scalar concentration normalised on the source concentration ensemble averaged over all source conditions (i.e., time-averaged over all 249 experimental runs). Dashed (red) curves mark the fits Eqs. (9)-(14) highlighting the ensemble-averaged flow envelopes and our estimate of the centerlines based on the contours of constant scalar concentration. For comparison, centerlines are marked as follows: $\triangle$ at the height $z=0.6$ for which $x_{\text {cling }} / x_{\text {free }}=1.32$, and $\bigcirc$ at $z=4.0$ for which $x_{\text {cling }} / x_{\text {free }}=1.18$. The marker - indicates the position along the centerline at which horizontal and vertical momentum fluxes are equal; to achieve this, clinging jets must travel laterally $20 \%$ (approx.) farther than free jets ( $x_{\text {cling }} / x_{\text {free }} \approx 5.5 / 4.5$ ).

light intensity; Sec. IV C) and envelope edges (obtained from ensemble averaging the time-averaged edges from each source condition; Sec. IV C) were fitted with polynomials of increasing order until the coefficient of determination between the experimental data and the polynomial exceeded 0.999 . For free jets

$$
z= \begin{cases}0.0047 x^{3}+0.0521 x^{2}-0.2675 x, & \text { upper edge } \\ 0.0048 x^{4}-0.0285 x^{3}+0.1163 x^{2}-0.0792 x, & \text { centerline } \\ 0.0494 x^{4}-0.2220 x^{3}+0.3844 x^{2}+0.0881 x, & \text { lower edge }\end{cases}
$$

For clinging jets

$$
z= \begin{cases}0.0495\left(x-x_{c}\right)^{3}+0.1451\left(x-x_{c}\right)^{2}+0.6697\left(x-x_{c}\right), & \text { upper edge } \\ 0.0189 x^{3}-0.0672 x^{2}+0.0557 x, & \text { centerline } \\ 0.0309 x^{4}-0.1577 x^{3}+0.2650 x^{2}-0.0959 x, & \text { lower edge }\end{cases}
$$




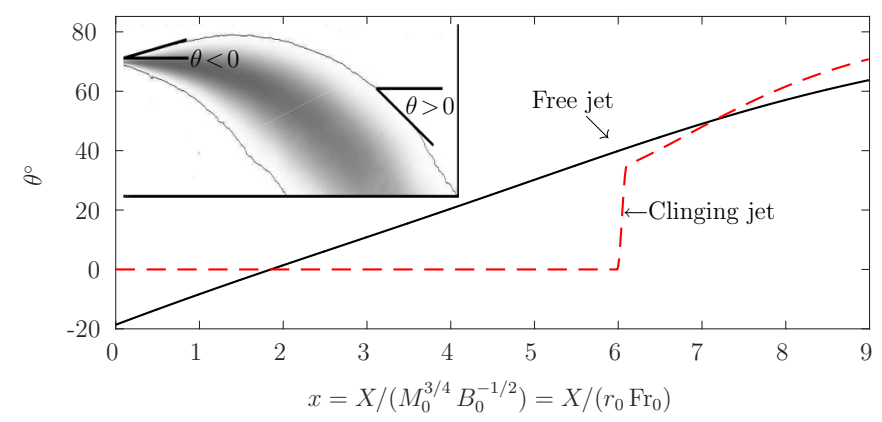

FIG. 8. The variation of the angle $\theta$ (see inset) between the horizontal and the tangent to the upper edge of the flow envelope for free and clinging jets. The upper limit of the vertical axis marks the angle between the horizontal and the edge of a vertically rising pure plume, assuming a top-hat entrainment coefficient of $\alpha=0.1 \sqrt{2}$, i.e., $\theta_{p} \approx 85^{\circ}$.

These polynomials, marked by dashed lines on Fig. 7, fit the data well for $z \lesssim 5$ and $x \geqslant 0$ [noting that Eq. (12) requires $x \geqslant x_{c}$, where $x_{c}\left(=\overline{X_{c}} / r_{0} \mathrm{Fr}_{0}\right)$ is the scaled mean cling length at high $\mathrm{Fr}_{0}$; we find $x_{c}$ reduces to $x_{c}=6.0$, see Eq. (15) and Sec. V B 2, in the high-Fr $\mathrm{F}_{0}$ limit].

A number of statistics can be used to compare the trajectories of free and clinging jets; see, for example, Sec. VB 1. For instance, there must exist a location within each jet at which the vertical momentum flux (induced by the action of the buoyancy force) is equal to the horizontal (conserved) momentum flux from the source. As we shall see, the location of this point, $\left(x_{M}, z_{M}\right)$, provides further insights. Assuming that the losses in clinging jets due to friction at the boundary are negligible (reasonable given the boundary is smooth), the relationship between the horizontal and vertical components of momentum flux requires that at $\left(x_{M}, z_{M}\right)$ the tangent to the centerline is at an angle of $45^{\circ}$ to the horizontal. Thus, $\left(x_{M}, z_{M}\right)$ can be uniquely identified from Eqs. (10) and (13). Our data indicate that $s_{M, \text { cling }} / s_{M \text {,free }}=1.21$ and $x_{M, \text { cling }} / x_{M \text {,free }}=1.22$, i.e., clinging jets travel approximately $20 \%$ farther (both laterally and based on the length of their centerline) than free jets to reach the point at which the vertical momentum flux equals the horizontal source momentum flux. To induce an identical vertical momentum flux, both jets would be expected to have traveled the same vertical distance. Our measurements indicate $z_{M, c l i n g} / z_{M, \text { free }}=0.993$, suggesting that our measurements might be accurate to within about $1 \%$.

It is notable that the envelopes for clinging jets show a broader spread for the edge detected furthest from the source [Fig. 7(b)]. This increased spread results from the widely pulsating behavior of the jet in this region (without good reason to do otherwise, the averaging times for the envelopes of free and clinging were chosen to be similar for our experiments). The spread apparent in the location of the edge of clinging jets, as the buoyancy force pulls the jet away from the boundary, is reflected in our measurements of the mean cling length, $\overline{X_{c}}$ (Sec. V B 2), and the source of this spread is discussed in Sec. IV.

\section{The variation in the envelope angle}

To contrast further the behaviors of free and clinging jets, it is informative to consider the variation of $\theta$ (see Fig. 8 inset) defining the angle between the horizontal and the tangent to the upper edge of the flow envelope. Figure 8 plots the variation in $\theta$ with horizontal distance from the source deduced from Eq. (9) for free jets and Eq. (12) for clinging jets. For free jets the vertical position of the envelope's upper edge increases (i.e., $\theta<0$ ), reaching a maximum at about two horizontal jet lengths $(x \approx 2)$ from the source. The rate of increase in $\theta$ is approximately constant over (approximately) six horizontal jet lengths from the source, after which the rate slows, indicating, as expected, that $\theta$ may only asymptotically approach the limit corresponding to a vertical pure plume (the upper limit of the vertical axis in Fig. 8 marks this asymptote, i.e., $\theta_{p} \approx 85^{\circ}$ ). 


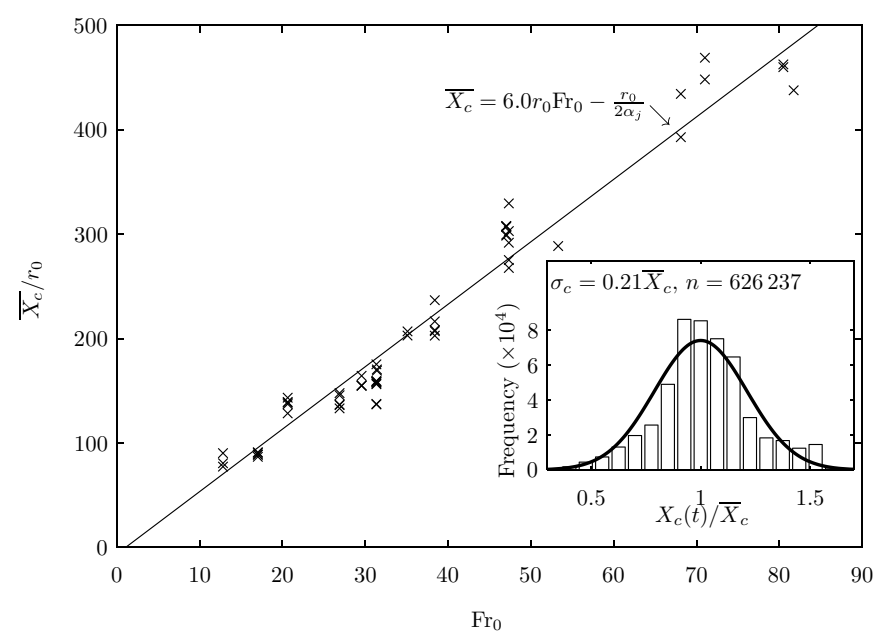

FIG. 9. Time-averaged cling length, $\overline{X_{c}} / r_{0}$, vs source Froude number $\operatorname{Fr}_{0}$. The line marks the best fit (coefficient of determination $R^{2}=97.2 \%$ ) of $\overline{X_{c}}$ to the momentum jet length, $r_{0} \mathrm{Fr}_{0}=M_{0}^{3 / 4} / B_{0}^{1 / 2}$, assuming a virtual origin equal to that of a pure jet. Inset shows a histogram of the (normalized) instantaneous cling length $X_{c}(t) / \overline{X_{c}}$ with the measured standard deviation, $\sigma_{c}$, and number of observations, $n$, marked. For comparison, the Gaussian with a mean of unity and standard deviation of $\sigma=0.21$ is overlaid.

For clinging jets, $\theta=0$, while the flow remains attached to the horizontal boundary, i.e., for $x \leqslant 6$. At $x=6$ there is a step increase to $\theta \approx 35^{\circ}$, an angle some $5^{\circ}$ less than for free jets at the same horizontal position. Note the vertical position of the envelope edges of free and clinging jets differ substantially at $x=6$ (for clinging jets $z \approx 0$ and for free jets $z \approx 1.3$ ). The angle $\theta$ then increases rapidly, slightly exceeding the envelope angle of free jets for $x \gtrsim 7$.

\section{Cling length: The length of the attachment for clinging jets}

Figure 9 plots the time-averaged dimensionless cling length against source Froude number and indicates that cling length increases approximately linearly with jet length. Assuming a horizontal offset of $X_{v o}=r_{0} / 2 \alpha_{j}$ Eq. (5), where $\alpha_{j}=0.055 \sqrt{2}$ as discussed in Sec. IV, the best fit to our data gives the cling length as

$$
\overline{X_{c}}=6.0 r_{0} \mathrm{Fr}_{0}-X_{v o},
$$

with a reasonable coefficient of determination, $R^{2}=97.2 \%$. The fluctuation in cling length is illustrated by the histogram of (normalised) instantaneous cling lengths, $X_{c}(t) / \overline{X_{c}}$ inset within Fig. 9 . The standard deviation in this measurement is $21 \%$, with approximately $29 \%$ of the data lying beyond one standard deviation of the mean cling length. This highlights the dramatic fluctuations in cling length that occur (see the observations of Ref. [25]) as large-scale eddies pulse through. These fluctuations may account for the larger uncertainty observed in the measurements; for example, taking the 10 data points available for notionally similar $\operatorname{Fr}_{0}\left(46 \leqslant \mathrm{Fr}_{0} \leqslant 48\right)$ the standard deviation is approximately $6 \%$ of the mean. This variation is in line with the uncertainty estimates discussed in Sec. IV D.

Our findings for the time-averaged cling length, Eq. (15), are slightly at odds with the findings of Ref. [25] for which a fit of the same form as Eq. (15) yields a constant of proportionality of approximately 4.5. It is not clear whether this difference arises due to differences in the experimental setups or in defining and detecting the instantaneous cling length. Of note is that the lateral location of the centerline's departure from the proximity of the boundary occurs at approximately the same location. 


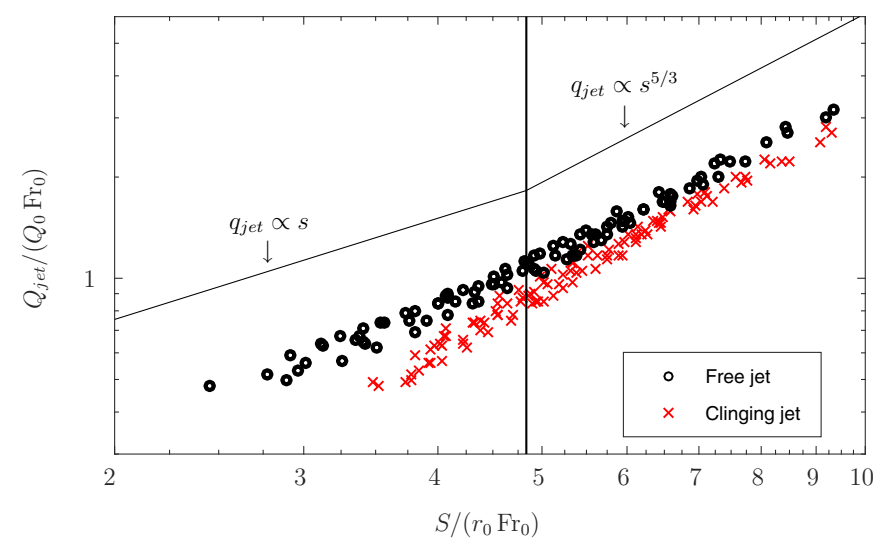

FIG. 10. The variation in the volume flux across horizontal planes, $Q_{\text {jet }} /\left(Q_{0} \mathrm{Fr}_{0}\right)$, with distance along the jet centerline, $S /\left(r_{0} \mathrm{Fr}_{0}\right)$. The vertical line marks the point $s=s_{M}$. The classic power laws for pure jets $(q \propto s)$ and pure plumes $\left(q \propto s^{5 / 3}\right)$ are marked by the solid lines.

\section{Entrainment and the variation in the volume flux $Q_{\text {jet }}$ with centerline distance}

From our estimates for the coordinates of the jet centerline, Eqs. (10) and (13), we establish a coordinate, $S$, that indicates position along the centerline measured from the source at $S=0$. Figure 10 plots on log-log axes the variation in our measurements of the volume flux across horizontal planes $Q_{j e t} /\left(Q_{0} \mathrm{Fr}_{0}\right)$ with the normalized coordinate, $s=S /\left(r_{0} \mathrm{Fr}_{0}\right)$. Clearly the figure does not provide quantitative estimates of the volume flux in the jets across planes perpendicular to the centerline, since we measured only the volume flux across horizontal planes and could only identify the centerline a posteriori. Figure 10 is included solely to shed light on the results presented in Sec. V A, by way of illustrating our measurements of volume flux relative to a distance which is characteristic of the average distance traveled by jet fluid crossing the given horizontal plane; we selected the centerline as a characteristic indication of the distance traveled. The data show that clinging jets travel farther to entrain the same volume flux as free jets. This confirms, as expected, that the presence of the boundary does indeed (locally) reduce the entrainment per unit distance traveled by clinging jets. However, the diversion of the jet trajectory by the boundary is substantial (Sec. V B) so that, at moderate vertical distances from the source, clinging jets have traveled significantly farther than equivalent free jets. The increased distance traveled by clinging jets is sufficient to ensure that, despite the reduction in entrainment per unit distance traveled, the total volume flux they entrain exceeds that entrained by an equivalent free jet.

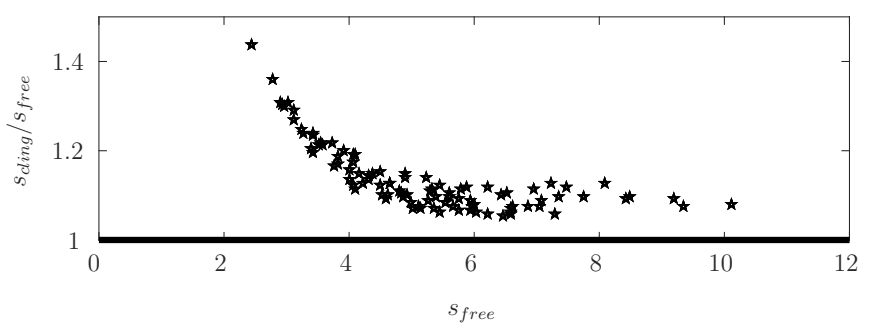

FIG. 11. The variation in the ratio of the centerline distance traveled by clinging jets and free jets to attain an equal volume flux, $s_{\text {cling }} / s_{\text {free }}$, plotted against the distance traveled by free jets, $s_{\text {free }}$. The horizontal axis marks $s_{\text {cling }} / s_{\text {free }}=1$. 
Figure 11 shows the variation of $s_{\text {cling }} / s_{\text {free }}$ with the distance traveled by the free jet. The data confirm that close to the source, $s_{\text {free }} \lesssim 3$, a clinging jet has to travel approximately $40 \%$ farther to attain the same volume flux as an equivalent free jet. At greater distances from the source the ratio drops rapidly and then more slowly, supporting the assertion that the far-field trajectories of free and clinging jets are similar.

\section{CONCLUSIONS}

The role of a planar horizontal boundary on the dynamics of an otherwise horizontal free jet of buoyant fluid was examined, with a focus on turbulent high Froude number $\left(\mathrm{Fr}_{0} \gtrsim 12\right)$ incompressible jets. Scaling the source-boundary separation on the source jet length we find that the jet is unaffected by the boundary for dimensionless separations of $z_{0} \gtrsim 0.7$ and that for $z_{0} \lesssim 0.2$ the jet attaches to and clings to the boundary, the length of the cling scaling linearly on the jet length (15). For source-boundary separations between those yielding the universal behavior of free jets or of clinging jets (i.e., for $0.2 \lesssim z_{0} \lesssim 0.7$ ), we observed intermittent attachment and detachment of the jets, a behavior not well reflected by time-averaged statistics.

Our scaled data identify universal flow envelopes (jet perimeters) for free and clinging jets. Measurements of volume flux identify vertical regions where the volume flux in a clinging jet exceeds an equivalent free jet. A simple parametrization of the ratio of the velocity and buoyancy widths enables good prediction of the volume fluxes using an existing model. However, this model fails to accurately predict the jet trajectory. We provide polynomial approximations for the jet centerlines and bounding flow envelopes to simplify the validation of future advances in the modeling of both free and clinging jets.

Our measurements of the evolution of the volume flux could enable a horizontal buoyant jet to be better tailored to suit a particular application. A clinging jet entrains less (than an equivalent free jet) per unit distance traveled; however, it travels horizontally farther while remaining close to the plane of the source so that in a vertical coordinate the volume flux in the clinging jet is actually increased. A free jet becomes a clinging jet simply with the introduction of a horizontal boundary near the source and the scalar quantities in the flow (e.g., pollutants) are then advected farther horizontally and diluted to lower concentrations while remaining close to the plane of the source. Thus, the flow established in the application of a free jet could be relatively easily altered by instead establishing a clinging jet. Horizontal buoyant jets are frequently formed within environments which may be stratified or that may not be entirely quiescent, but our findings still hold relevance as we demonstrate in Appendix A.

\section{ACKNOWLEDGMENT}

The authors gratefully acknowledge support and funding from Dyson Technology Ltd.

\section{APPENDIX A: TWO EXAMPLE APPLICATIONS OF HORIZONTAL BUOYANT JETS IN QUIESCENT UNIFORM ENVIRONMENTS}

Discharges into the oceans and other bodies of open-water frequently occur through outfalls. These are substantial pipes along which ports (or holes) are positioned, through which the discharge is forced. Discharges from outfalls are aqueous solutions, often with density differences in the range $0.1 \% \leqslant\left|\rho_{0}-\rho_{e}\right| / \rho_{e} \leqslant 3.5 \%$, the resulting jet being either positively or negatively buoyant. Data from Ref. [6] give source Froude numbers in the range $11 \lesssim \operatorname{Fr}_{0} \lesssim 200$ with corresponding jet lengths of $84 \mathrm{~cm} \lesssim L_{M} \lesssim 1500 \mathrm{~cm}$. Moreover, the characteristic time scale (over which the source buoyancy flux induces a momentum flux proportional to the source momentum flux) is $5 \mathrm{~s} \lesssim T_{M}=M_{0} / B_{0} \lesssim 600 \mathrm{~s}$. Typical time scales for the buoyancy frequency in lakes or oceans are in the range $30 \mathrm{~s} \lesssim N^{-1} \lesssim 100 \mathrm{~s}$ [26], where $N^{2}=-g d \hat{\rho_{a}}(z) / d z$, and $\hat{\rho_{a}}(z)$ is the normalized local ambient density. The ratio of the time scales, $T_{M} / N^{-1}$, provides an indication of whether the source 
momentum flux might be altered more rapidly with height by the effects of the source buoyancy flux $\left(T_{M} / N^{-1} \gg 1\right.$, as is clearly the case in the uniform environments considered herein) or by the effects of the stratification $\left(T_{M} / N^{-1} \ll 1\right)$. The data of Ref. [6] indicate $0.05 \lesssim T_{M} / N^{-1} \lesssim 20$ and suggest that for certain discharges the dynamics may be affected only by the stratification relatively far (temporally or spatially) from the source. Velocities in coastal oceans may be regarded to be in the range $10 \mathrm{~cm} / \mathrm{s} \lesssim V_{a} \lesssim 100 \mathrm{~cm} / \mathrm{s}$ [38], and hence the ratio of the inertial velocity scale in the oceans to that at the source of the discharges falls in the range $0.02 \lesssim V_{a} / V_{0} \lesssim 0.5$. This suggests that, for discharges into many lakes or oceans, the effects of source momentum flux will likely dominate the effects of the inertia in the ambient environment. As a consequence, studies of these releases into quiescent environments of uniform density (as is the case herein) may provide useful insights.

The understanding of horizontal buoyant jets is fundamental elsewhere, for example, in determining how the flow established by a domestic fan heater might warm a room. Source Froude numbers for fan heaters broadly range within $0.5 \lesssim \mathrm{Fr}_{0} \lesssim 50$, with corresponding jet lengths being $10 \mathrm{~cm} \lesssim L_{M} \lesssim 200 \mathrm{~cm}$ and time scales $0.2 \mathrm{~s} \lesssim T_{M} \lesssim 5 \mathrm{~cm}$. Within buildings, the heating and ventilation systems are ideally designed to avoid uncomfortable draughts. Recommended velocities in the ambient are therefore small, e.g. $V_{a} \approx 10 \mathrm{~cm}$ [39] provides $0.01 \lesssim V_{a} / V_{0} \lesssim 0.3$. Temperature differences over the height of a room are typically no more than a few degrees Celsius, say, in the range 1 to $5{ }^{\circ} \mathrm{C}$, and taking room heights in the range $250 \mathrm{~cm} \lesssim H \lesssim 400 \mathrm{~cm}$ yields typical relative stratification strengths of between $0.7 \lesssim T_{M} / N^{-1} \lesssim 55$. Therefore, the dynamics of the flow arising from a fan heater within a domestic room may often be reasonably approximated by consideration of the flow within a quiescent environment of uniform density.

\section{APPENDIX B: OBTAINING ESTIMATES OF THE FLOW ENVELOPES}

Within each image the instantaneous edges of the jet were identified (using the Canny edge detection algorithm provided in Matlab [40]). The location of the outermost edges were timeaveraged over each experimental run (a "run" referring to the time between iterative adjustments to $Q_{\text {out }}$ ) to provide an estimate of the flow envelope of the jet as in Fig. 3. However, to obtain better estimates of the flow envelope we desired to maximize the duration over which the location of the edges were time-averaged. In any given image the edges could be identified only within the portion of the image above the interface. For each set of source conditions $\left(\pi Q_{0}, r_{0}, g_{0}^{\prime}\right)$, the vertical position of the interface was systematically altered (through the iterative adjustments of $Q_{\text {out }}$ to obtain the variation in volume flux with height) and, hence, the portion of the image within which the edges could be detected also varied. Estimates of the flow envelopes were produced, for each set of source conditions, by averaging the location of the edges depending on the number of experimental runs providing data for that pixel, weighted by the corresponding recording duration. Figure 3(a) shows an instantaneous image of a jet with the vertical position of the interface as far below the source as was possible given the source condition, in this case $Q_{\text {out }}=70 Q_{0}$. Figure 3(b) shows the estimate of the flow envelope; note that near the source the envelope was averaged over all experimental runs for the given source condition, in this case nine experimental runs. Farthest from the source, the estimate of the envelope could only be obtained by averaging over a single experimental run.

[1] F. P. Ricou and D. B. Spalding, Measurements of entrainment by axisymmetrical turbulent jets, J. Fluid Mech. 11, 21 (1961).

[2] G. N. Abramovich, The Theory of Turbulent Jets (MIT Press, Cambridge, MA, 1963).

[3] B. R. Morton, G. Taylor, and J. S. Turner, Turbulent gravitational convection from maintained and instantaneous sources, Proc. R. Soc. London A 234, 1 (1956).

[4] B. R. Morton, Forced plumes, J. Fluid Mech. 5, 151 (1959). 
[5] G. R. Hunt and N. B. Kaye, Lazy plumes, J. Fluid Mech. 533, 329 (2005).

[6] R. C. Y. Koh and N. H. Brooks, Fluid mechanics of waste-water disposal in the ocean, Annu. Rev. Fluid Mech. 7, 187 (1975).

[7] M. B. Glauert, The wall jet, J. Fluid Mech. 1, 625 (1956).

[8] B. E. Launder and W. Rodi, The turbulent wall jet: Measurements and modeling, Annu. Rev. Fluid Mech. 15, 429 (1983).

[9] D. J. Tritton, Physical Fluid Dynamics (Oxford Science Publications, Oxford, 1988).

[10] Y. B. Zel'dovich, The asymptotic laws of freely-ascending convective flows, Zhur. Eksper. Teor. Fiz. 7 (in Russian), English translation in Selected Works of Yakov Borisovich Zel'dovich, Vol. 1, Chemical Physics and Hydrodynamics, edited by J. P. Ostriker, G. I. Barenblatt, and R. A. Sunyaev (Princeton University Press, Princeton, 1992).

[11] G. Abraham, Horizontal jets in stagnant fluid of other density, J. Hydraulics Div. Proc. ASCE 91, 138 (1965).

[12] L. N. Fan and N. H. Brooks, Discussion of "Horizontal jets in stagnant fluid of other density", by Gerrit Abraham, J. Hydraulics Div. Proc. ASCE 92, 423 (1966).

[13] L. N. Fan, Turbulent buoyant jets into stratified or flowing ambient fluids, Ph.D. thesis, California Institute of Technology, 1967.

[14] J. H. W. Lee and V. Cheung, Generalized Lagrangian model for buoyant jets in current, J. Environ. Eng. 116, 1085 (1990).

[15] G. H. Jirka, Integral model for turbulent buoyant jets in unbounded stratified flows. Part I: Single round jet, Environ. Fluid Mech. 4, 1 (2004).

[16] P. C. Yannopoulos and A. A. Bloutsos, Escaping mass approach for inclined plane and round buoyant jets, J. Fluid Mech. 695, 81 (2012).

[17] B. S. Ryskiewich and L. Hafetz, An experimental study of the free surface effect on a buoyant jet, Tech. Rep. U440-74-103, General Dynamics Corp. (1975).

[18] J. B. Riester, R. A. Bajura, and S. H. Schwartz, Effects of water temperature and salt concentration on the characteristics of horizontal buoyant submerged jets, ASME J. Heat Transfer 102, 557 (1980).

[19] R. J. Sobey, A. J. Johnston, and R. D. Keane, Horizontal round buoyant jet in shallow water, J. Hydraul. Eng. 114, 910 (1988).

[20] M. J. Davidson, M. Knudsen, and I. R. Wood, The behavior of a single, horizontally discharged, buoyant flow in a non-turbulent coflowing ambient fluid, J. Hydraul. Res. 29, 545 (1991).

[21] G. A. Kikkert, Buoyant jets with two and three-dimensional trajectories, Ph.D. thesis, University of Canterbury, Christchurch, New Zealand, 2006.

[22] S. N. Michas and P. N. Papanicolaou, Horizontal round heated jets into calm uniform ambient, Desalination 248, 803 (2009).

[23] A. J. Johnston and R. E. Volker, Round buoyant jet entering shallow water, J. Hydraul. Res. 31, 121 (1993).

[24] B. G. Newman, in The deflection of plane jets by adjacent boundaries: Coanda effect, Boundary Layer and Flow Control, Vol. 1, edited by V. G. Lachmann (Pergamon Press, New York, 1961), pp. 232-264.

[25] J. J. Sharp and B. D. Vyas, The buoyant wall jet, in Proceedings of the Institute of Civil Engineers, part 2, edited by Thomas Telford (ICE Publishing, 1977), Vol. 63, pp. 593-611.

[26] H. B. Fischer, E. J. List, R. C. Y. Koh, J. Imberger, and N. H. Brooks, Mixing in Inland and Coastal Waters (Academic, San Diego, 1979).

[27] I. K. Madni and R. H. Pletcher, Buoyant jets discharging nonvertically into a uniform, quiescent ambient: A finite-difference analysis and turbulence modeling, ASME J. Heat Transfer 99, 641 (1977).

[28] G. R. Hunt and H. C. Burridge, Fountains in industry and nature, Annu. Rev. Fluid Mech. 47, 195 (2015).

[29] W. D. Baines, A technique for the direct measurement of volume flux of a plume, J. Fluid Mech. 132, 247 (1983).

[30] C. Cenedese and S. B. Dalziel, Concentration and depth field determined by the light transmitted through a dyed solution, in Proceedings of the 8th International Symposium on Flow Visualization, Vol. 8 (1998), pp. 1-37.

[31] D. M. Allgayer and G. R. Hunt, On the application of the light-attenuation technique as a tool for non-intrusive buoyancy measurements, Exp. Thermal Fluid Sci. 38, 257 (2012). 
[32] G. F. Lane-Serff, P. F. Linden, and M. Hillel, Forced, angled plumes, J. Hazard. Mater. 33, 75 (1993).

[33] J. S. Turner, Turbulent entrainment: The development of the entrainment assumption, and its application to geophysical flows, J. Fluid Mech. 173, 431 (1986).

[34] A. Ezzamel, P. Salizzoni, and G. R. Hunt, Dynamical variability of axisymmetric buoyant plumes, J. Fluid Mech. 765, 576 (2015).

[35] P. N. Papanicolaou and E. J. List, Investigations of round vertical turbulent buoyant jets, J. Fluid Mech. 195, 341 (1988).

[36] N. R. Panchapakesan and J. L. Lumley, Turbulence measurements in axisymmetric jets of air and helium. Part 2: Helium jet, J. Fluid Mech. 246, 225 (1993).

[37] G. A. Kikkert, M. J. Davidson, and R. I. Nokes, Buoyant jets with three-dimensional trajectories, J. Hydraul. Res. 48, 292 (2010).

[38] G. T. Csanady, Circulation in the Coastal Ocean (Springer, New York, 1982).

[39] P. O. Fanger and N. K. Christensen, Perception of draught in ventilated spaces, Ergonomics 29, 215 (1986).

[40] J. Canny, A computational approach to edge detection, IEEE Transactions on Pattern Analysis and Machine Intelligence, no. 6 (IEEE, 1986), pp. 679-698. 